\title{
Iran, automne 2009
}

Françoise Verrey Bass

Correspondance:

Dr Françoise Verrey Bass Plänkestrasse 12

CH-2502 Bienne

fraverrey[at]gmx.ch
Le voyage en Iran a débuté en fait à Istanbul: se trouver un vêtement correct selon les normes exigées en Iran pour pouvoir se promener en dehors de sa chambre d'hôtel! Donc à porter partout et tout le temps pendant une semaine. Le chauffeur de taxi, au lieu de nous montrer les superbes œuvres architecturales de la ville, nous a emmenées, ma sœur et moi, dans un quartier reculé du souk, un petit magasin en couloir, à l'étage, où une gracieuse stambouliote nous a fait essayer des sortes de manteaux nous recouvrant les genoux. Pour 50 euros nous avons trouvé ce qu'il fallait: un manteau ne collant pas au corps, sobre, bleu foncé. Seul désavantage; certainement trop chaud.

Arrivées au poste frontière de l'Iran au sud-est du Turkménistan nous enfilons le manteau et nous nous couvrons la tête avec le foulard bleu apporté de Suisse.

Toutes les femmes du groupe font de même, tandis que les hommes, ces veinards, peuvent rester en manches de chemises et pantalons légers. Pas de shorts, évidemment.

Il fait un doux temps de fin d'été, mais toujours encore assez chaud pour nous.
Le passage de la frontière en Iran se fait sans encombre, hommes et femmes en faction, tous jeunes et aimables, avec de grands sourires étincelants sur des dents blanches et pour les hommes des cheveux couleurs ailes de corbeau - chez les femmes les cheveux sont bien sûr cachés par un foulard entourant strictement l'ovale du visage. Nous pouvons boire de l'eau, aller aux toilettes, nous promener un peu dehors à l'ombre en attendant l'arrivée au poste frontière du nouveau bus. Quelle différence avec l'entrée au Turkménistan!

Départ à travers un paysage très montagneux direction Mashad, grand lieu de pèlerinage des Chiites iraniens au nord-est de l'Iran. Au programme visite de la mosquée et conversation avec un Imam. A la descente du bus chaque femme reçoit un tchador à passer par-dessus ses habits. Le chauffeur du bus, un grand et beau gaillard aux cheveux bouclés nous aide à passer ce tissu très léger, noir, couvert de fleurs blanches, par-dessus la tête et à le crocher derrière la nuque. Il nous montre comment le tenir fermé d'une main devant la poitrine et en avant dans la foule. Les femmes d'un côté, les hommes de

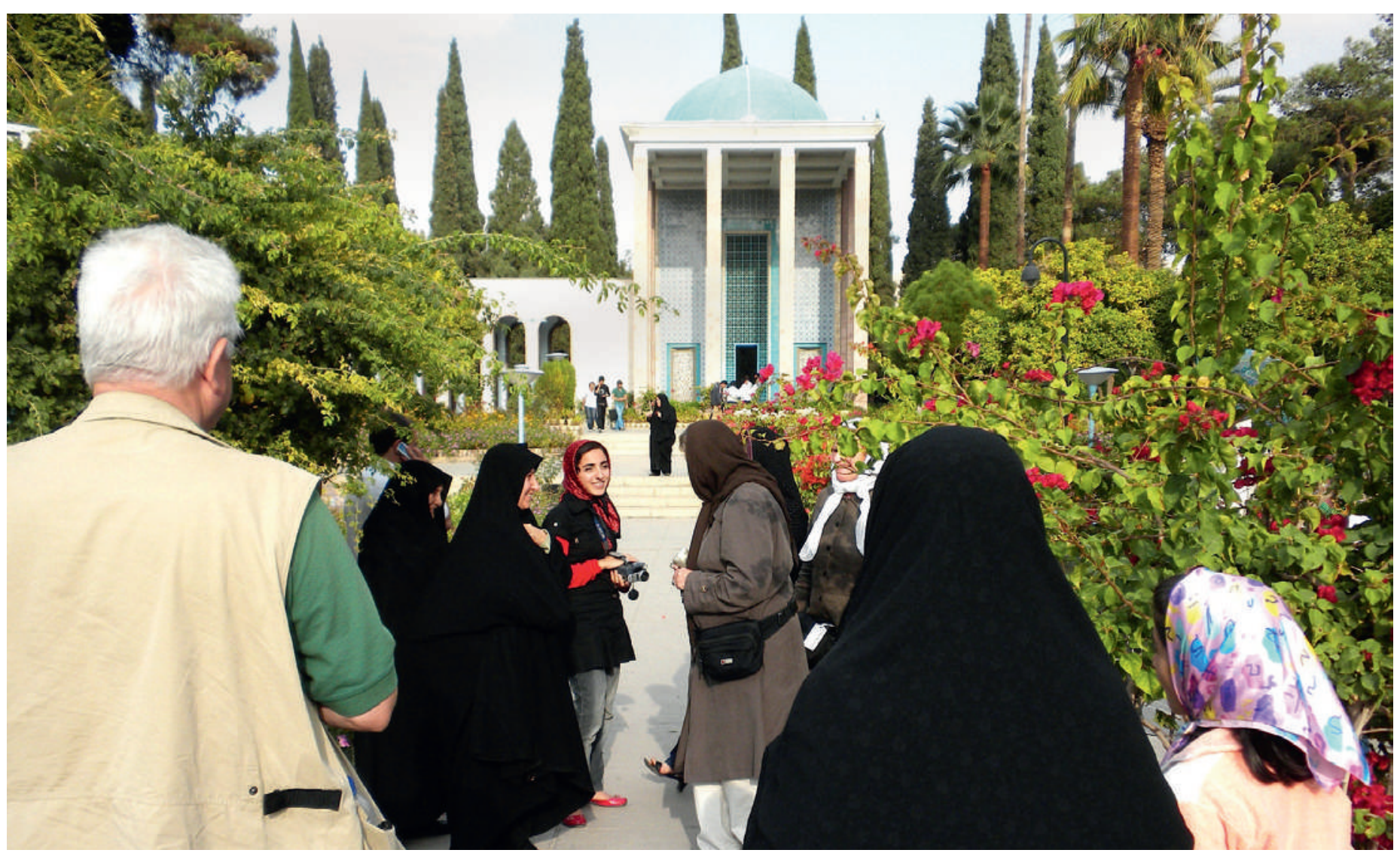

Début du voyage en Iran: se trouver un vêtement correct pour pouvoir se promener en dehors de sa chambre d’hôtel. 


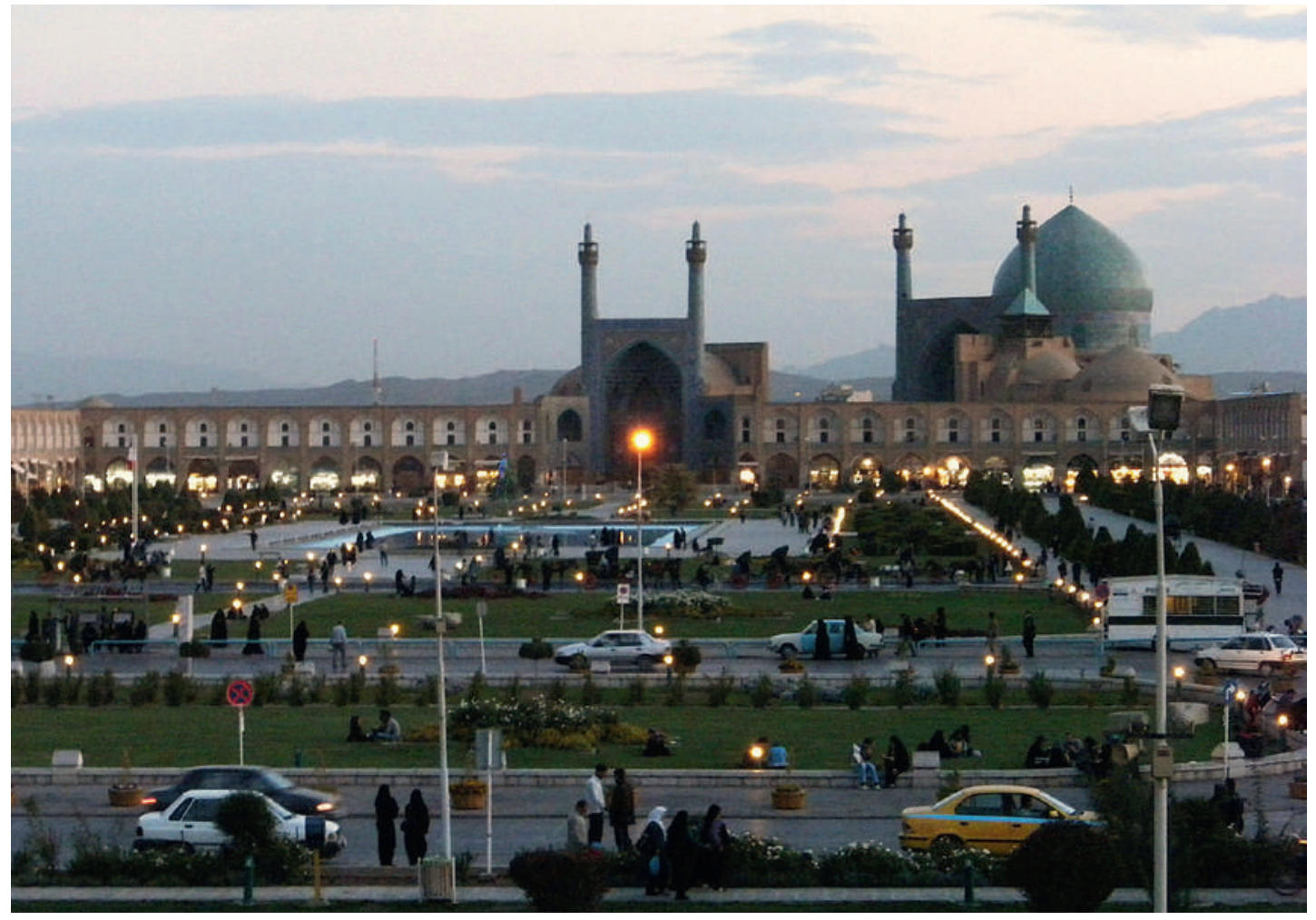

Place Naqsh-e Jahan à Ispahan.

l'autre. Nous nous engouffrons par une petite porte dans un hall d'entrée étroit et pas très haut, mal éclairé, et là, quelque chose bouche le passage plus loin. Le hall est rempli, les femmes entrées derrière nous nous poussent en avant. Debout parmi elles, en tchador noir pour la plupart, dans cette pénombre, il faut faire très attention à ne pas tomber. Des petits cris fusent de tous parts. Une enfant de peut-être 5 ans se met à pleurer à côté de moi. Entourée de femmes,

\section{Téhéran: avec nos manteaux, nous avons vraiment l'air}

\section{de ploucs venus de la campagne.}

ne voyant que des «murs noirs», elle doit paniquer. Sa mère la prend dans ses bras et nous avançons d'un pas. Puis un autre pas, et ainsi de suite. Pour arriver jusqu'à une barrière avec deux ouvertures. Devant chaque ouverture, barrant le passage, une femme de la sécurité. J'ai depuis longtemps perdu mes compagnes de route. La jeune femme devant moi commence à me palper de la tête aux pieds. Arrivée au ventre, elle sent ma poche de voyage. Elle arrête sa palpation et me regardant dans les yeux avec un sourire dans les siens: «Tourist?» demande-t-elle. J'acquiesce de la tête. Alors elle me fait passer sans palper plus loin et l'obscurité du couloir me crache dans une cour pleine de soleil où attendent les hommes du groupe. «Enfin» leurs commentaires!
Une belle, immense mosquée, un complexe de mosquées. On nous explique: ici les cuisines, là les dortoirs, plus loin l'infirmerie, la bibliothèque, l'habitat des religieux, un vrai village, ou plutôt une communauté fermée accueillant les pèlerins de passage, les nourrissant, les soignant si nécessaire.

L'imam nous attend. Notre guide dans cette mosquée insiste, il n'a qu'une demi-heure à nous consacrer. Majestueux et bien qu'assis sur une chaise il nous salue en nous regardant de haut. Première question brève à laquelle il répond en long et en large, en anglais, s'étendant abondamment sur des points absolument inintéressants. 10 minutes de passées. Deuxième question, posée par ma sœur: «D'après le Coran, homme et femme sont égaux. Par contre d'après la Charia, les femmes n'ont que la moitié de la valeur des hommes (exemples: le témoignage d'une femme devant un tribunal ne vaut que la moitié de celui d'un homme. Ou la fille n'hérite que la moitié de ce qu'hérite le garçon). Comment est-il dès lors possible de concilier Coran et Charia?» Réponse: «La femme est faible, elle doit être protégée par l'homme, c'est pour cela qu'il doit avoir les moyens de la protéger. La femme a moins de force et d'endurance que l'homme... Par exemple: études de médecine, salle d'autopsie, la femme ne tient pas le coup et tombe régulièrement sans connaissance...» Gros éclats de rire et remarques à haute voix dans notre groupe, nous ne comprenons pas du tout le choix de cet exemple. Et de plus je me sens obligée de constater que les seuls que j'ai vus tomber en salle d'autopsie, c'était 2 étudiants, mais pas d'étudiantes. 


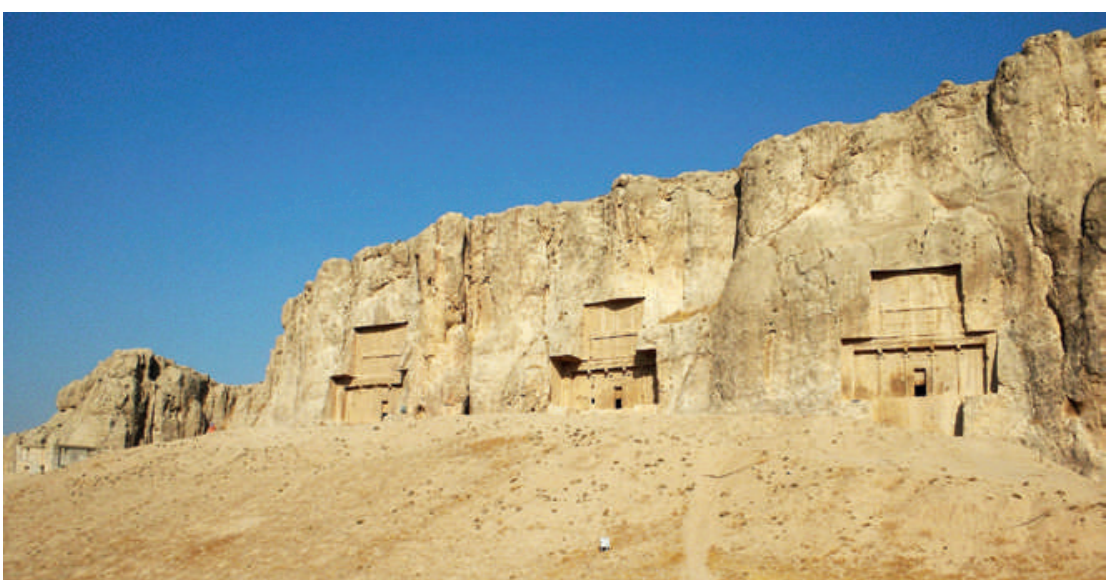

Tombeaux royaux de Naqsh-e Rostam.

20 minutes sont passées, fini, les questions sont terminées, l'imam, tout à coup impatient n'a plus le temps, il doit se rendre à la prière de 11 heures.

Nous nous retrouvons près du bus et pouvons enlever ce tchador qui, si léger qu'il soit, a augmenté la chaleur nous accompagnant sans répit.

Notre jeune guide en Iran est étudiant, très sympathique. Il aime son pays et nous décrit avec beaucoup de ferveur ses richesses archéologiques et culturelles. Mais dans le bus, il nous parlera souvent à mots couverts de l'oppression du peuple, surtout des intellectuels. Lui, il veut finir ses études sans avoir de problème avec la police, en évitant tout rassemblement et surtout manifestation, pour ne pas être dans un fichier quelconque. Ses études terminées, il demandera un visa pour les Etats-Unissi possible, et... La fuite des cerveaux.

Mais dans le bus, il nous parlera souvent à mots couverts de l'oppression du peuple, surtout des intellectuels.

A Téhéran nous admirons les tenues élégantes et quelquefois fort courtes et moulantes de beaucoup de jeunes femmes. Avec nos manteaux, nous avons vraiment l'air de ploucs venus de la campagne, sauf que les campagnardes portent le long tchador noir dans le fond bien plus élégant et ont peut-être moins chaud que nous, quoique, ce sont aussi elles qui portent le voile intégral. Ma sœur, qui n'en est pas à son premier voyage en Iran, fait la remarque que curieusement le voile montre depuis le début du siècle une sérieuse tendance à glisser vers l'arrière tête. On voit de plus en plus de cheveux, surtout chez les jeunes femmes. Tandis que nous portant le voile placé correctement, nous constatons que nous entendons un peu moins bien, mais surtout que notre champ visuel latéral est diminué, nous sommes obligées de bouger beaucoup plus la tête quand nous voulons, par exemple, traverser une route. Pendant les longs trajets en bus en dehors des villes nous pouvons enlever le voile et le manteau et sommes ainsi un peu plus libre de nos mouvements. Le guide est sur ses gardes et nous avertit à temps pour ne pas avoir d'ennuis.

De Téhéran nous partons en avion pour Shiraz, la ville des roses et des rossignols à $1600 \mathrm{~m}$ d'altitude, au climat agréable, à la végétation luxuriante. C'est aussi la ville des poètes Saadi et Hafis, les maîtres de la poésie persane. Nous allons visiter leur tombe. Là, nous comprenons l'amour des persans pour la poésie. Il y a foule sur les deux esplanades, des familles entières viennent rendre hommage à leurs grands poètes, restent respectueuses, silencieuses devant les tombes dans ces grands jardins verdoyants et très fleuris. Même les enfants calment leur exubérance et leurs jeux dans les pavillons.

Partout où nous passons des jeunes adultes nous accostent et nous posent nombre de questions sur notre vie en Europe, mais aussi sur notre opinion concernant leur pays. Nous faisons très attention dans nos réponses. Mais cette curiosité de la génération des 20 à 40 ans nous a beaucoup frappé.

Le lendemain nous avons rendez-vous avec le passé, les ruines de Persepolis. Un tout autre monde, mais le reflet des fresques admirées reste ancré dans la mémoire ainsi que les ruines de Pasargadae, la première capitale perse, que nous avons vue sur le hautplateau du massif de Zagros le jour suivant.

Nous arrivons à la tombée de la nuit à Ispahan, au Palais Abbasi, le plus merveilleux hôtel de la ville, un ancien caravansérail. Même si nos chambres ne donnent pas sur le jardin, mais sur une petite cour latérale, l'impression de palais des mille et une nuits demeure. Féerique, surtout le soir quand tout est illuminé. Le plus beau souvenir de cette ville? Assise sur une terrasse au premier étage de la très connue maison de thé à l'entrée du bazar à regarder le soir envahir l'immense place autour de laquelle toute la ville s'articule, admirant l'eau irisée des jets d'eau qui en retombant fait frissonner la surface des immenses pièces d'eau. Le bleu qui règne sur toute la place s'intensifie avec la nuit qui arrive, les lumières s'allument un peu partout, donnant de nouvelles couleurs ocre aux mosquées, palais et arcades qui l'entourent. Le joyeux brouhaha du souk tout proche et les sabots des chevaux des calèches recouvrent le bruit des voitures... Quelques instants on oublie que c'est un autre temps aujourd'hui, on oublie la misère, la pauvreté, l'oppression surtout, on se laisse aller à la beauté et au charme du Moyen Orient.

Le surlendemain, à peine dans l'avion, nous sommes bien soulagées d'enlever voile et manteau et de les fourrer au fond du sac de voyage. Le manteau partira avec le prochain sac de «vieux» vêtements pour une organisation caritative. Par contre, impossible d'oublier la douceur et les senteurs dont nous nous sommes imprégnées tout le long de cette longue route à travers le pays, la gentillesse des gens, la richesse de cette culture et la beauté de ces paysages arides ou de ces oasis explosant de verdure et de multiples fleurs. 